\title{
CAYLEY COLOR GRAPHS OF INVERSE SEMIGROUPS AND GROUPOIDS
}

\author{
Nándor Sieben
}

\begin{abstract}
The notion of Cayley color graphs of groups is generalized to inverse semigroups and groupoids. The set of partial automorphisms of the Cayley color graph of an inverse semigroup or a groupoid is isomorphic to the original inverse semigroup or groupoid. The groupoid of color permuting partial automorphisms of the Cayley color graph of a transitive groupoid is isomorphic to the original groupoid.
\end{abstract}

\section{INTRODUCTION}

A classic result of Frucht says that every finite group is the automorphism group of some graph. This result follows from the fact that the group of automorphisms of the Cayley color graph of a finite group is isomorphic to the original group. The proof of both of these results and a comprehensive discussion of the Cayley color graph can be found in [Whi] and [GT].

In this paper we generalize the notion of Cayley color graphs for inverse semigroups and groupoids. We show that if $T$ is an inverse semigroup or a groupoid, then the set of partial automorphisms of the Cayley color graph of $T$, with composition and inverse as the operations, is isomorphic to $T$. We also show that the groupoid of color permuting partial automorphisms of the Cayley color graph of a transitive groupoid $\mathcal{G}$ is isomorphic to the semidirect product of $\mathcal{G}$ and the subgroup of Aut $\mathcal{G}$ preserving the generators of $\mathcal{G}$.

I thank Marc Fabbri for his suggestions.

\section{Preliminaries}

An inverse semigroup is a semigroup $S$ with a unique adjoint $s^{*}$ for each $s \in S$ satisfying $s s^{*} s=s$ and $s^{*} s s^{*}=s^{*}$. Every inverse semigroup can be represented as partial bijections of a set. We say that $s \leq t$ if $s=s s^{*} t$. The set of idempotents $\left\{s s^{*} \mid s \in S\right\}$ of $S$ is a semilattice, with the partial order. Our reference for inverse semigroups is [Pet].

A groupoid $\mathcal{G}$ is a small category with inverses. That is $\mathcal{G}$ is a set with a subset $\mathcal{G}^{(2)}$, a product map $(x, y) \mapsto x y: \mathcal{G}^{(2)} \rightarrow \mathcal{G}$ and an inverse map $x \mapsto x^{-1}: \mathcal{G} \rightarrow \mathcal{G}$ such that:

(a) $(x y) z=x(y z)$ for all $(x, y),(y, z) \in \mathcal{G}^{(2)}$;

(b) $\left(x, x^{-1}\right) \in \mathcal{G}^{(2)}$ for all $x \in \mathcal{G}$ and $x^{-1}(x y)=y,(x y) y^{-1}=x$ for all $(x, y) \in \mathcal{G}^{(2)}$.

The set $\mathcal{G}^{(2)}$ is called the set of composable pairs. The domain and range maps $d, r: \mathcal{G} \rightarrow U$ are defined by $d(x)=x^{-1} x$ and $r(x)=x x^{-1}$ where $U=\left\{x x^{-1} \mid x \in \mathcal{G}\right\}$ is the set of units of $\mathcal{G}$. Every groupoid is the disjoint union of transitive groupoids, and every transitive groupoid is the direct product of a group $G$ and a trivial groupoid $A \times A$ [Muh]. More precisely a transitive groupoid $\mathcal{G}$ is isomorphic to a groupoid $A \times G \times A$ where $(d, h, c)$

2000 Mathematics Subject Classification: Primary 20M18, Secondary 20B25

Keywords: Cayley color graph, inverse semigroup, groupoid 
and $(b, g, a)$ are composable whenever $b=c$, in which case their product is $(d, h g, a)$. The inverse of $(b, g, a)$ is $\left(a, g^{-1}, b\right)$. The set $A$ can be chosen to be the unit space of $\mathcal{G}$ and the group $G$ is isomorphic to the isotropy subgroup $\mathcal{G}_{u}^{u}=\{x \mid d(x)=u=r(x)\}$ for any unit $u$ of $\mathcal{G}$. Our references for groupoids are [Bro,Ren].

Let $T$ be an inverse semigroup or a groupoid. A subset $\Delta$ of $T$ generates $T$ if every element of $T$ can be written as a finite product of elements of $\Delta$.

A color digraph is a possibly infinite directed graph with possible multiple edges and loops together with a color function defined on the set of edges.

\section{The CAYley COLOR GRAPH OF AN INVERSE SEMigroup}

In this section $S$ denotes an inverse semigroup and $\Delta$ a set of generators of $S$.

The Cayley color graph of a group does not have an arrow colored with the inverse of a generator. Every time we have an arrow with color $g$ joining $s$ and $s g$, we can imagine a reversed arrow with color $g^{-1}$ joining $s g$ and $s g g^{-1}=s$. For inverse semigroups, $s g g^{*}$ is not necessarily the same as $s$. So we may need to include both the arrows colored with $g$ and $g^{*}$.

Definition 3.1. The tail of a vertex $v$ in a color digraph is the set tail $(v)$ of vertices that can be reached by a finite directed walk starting at $v$. We say that $v$ is a head of its tail.

Note that a tail may have more than one head, and that a tail contains each of its heads.

Definition 3.2. The Cayley color graph $D_{\Delta}(S)$ of $S$ with respect to the generating set $\Delta$ is the color digraph with vertices $S$ and edges

$$
\{(s, g, s g) \mid s \in S, g \in \Delta\}
$$

such that the edge $e=(s, g, s g)$ connects $s$ to $s g$ and has color $g$.

Lemma 3.3. If $s$ is a vertex of $D_{\Delta}(S)$ then tail $(s)=\{s r \mid r \in S\}$.

Proof. If $t \in$ tail $(s)$ then $t$ can be reached from $s$ by a finite directed walk. If $g_{1}, \ldots, g_{n}$ are the colors of the edges in this walk then $t=s\left(g_{1} \cdots g_{n}\right)$.

Conversely if $t=s r$ then $r=g_{1} \cdots g_{n}$ for some $g_{1}, \ldots, g_{n} \in \Delta$ and so $t$ can be reached from $s$ along a directed walk on edges with colors $g_{1}, \ldots, g_{n}$.

A Cayley color graph is connected, that is, any vertex $t$ can be reached from any other vertex $s$ by an undirected walk since $t t^{*} s s^{*}=s s^{*} t t^{*}$ is in the tail of both $s$ and $t$. The following example shows that the Cayley color graph is not necessarily strongly connected. 
Example 3.4. The inverse semigroup $S=\left\{s, s^{*}, s^{*} s, s s^{*}, 0\right\}$ generated by $\Delta=\left\{s, s^{*}\right\}$ subject to the relation $s s=0$, has the following Cayley color graph:

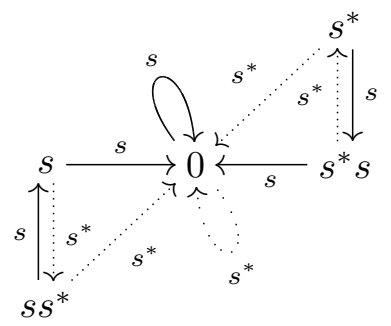

Note that $s^{*}$ cannot be reached from $s$ along a directed walk.

Example 3.5. The bicyclic semigroup [Pet, II.6.4] is the semigroup with identity element, generated by $\Delta=\{s, t\}$ subject to the relation $t s=1$. It has the following Cayley color graph:

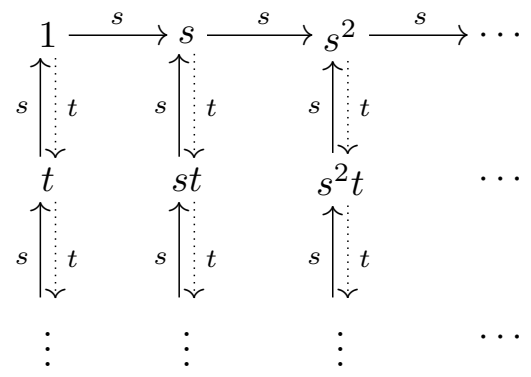

Definition 3.6. A partial automorphism of a Cayley color graph $D_{\Delta}(S)$ is a bijection $\alpha$ between two tails of $D_{\Delta}(S)$, such that $\alpha(s g)=\alpha(s) g$ for all $s \in \operatorname{dom}(\alpha)$ and $g \in \Delta$.

A partial automorphism is roughly a color preserving isomorphism between two subgraphs induced by tails.

Lemma 3.7. If $\alpha$ is a partial automorphism of $D_{\Delta}(S)$ then $\alpha(s r)=\alpha(s) r$ for all $s \in$ $\operatorname{dom}(\alpha)$ and $r \in S$.

Proof. Since $\operatorname{dom}(\alpha)$ is a tail, $s \in \operatorname{dom}(\alpha)$ implies $s r \in \operatorname{dom}(\alpha)$. There are $g_{1}, \ldots, g_{n} \in \Delta$ with $r=g_{1} \cdots g_{n}$. Hence

$$
\alpha(s r)=\alpha\left(s g_{1} \cdots g_{n}\right)=\alpha\left(s g_{1} \cdots g_{n-1}\right) g_{n}=\cdots=\alpha(s) g_{1} \cdots g_{n}=\alpha(s) r .
$$

Lemma 3.8. If $s$ is a vertex of $D_{\Delta}(S)$ then $s s^{*}$ is the unique idempotent for which tail $(s)=$ tail $\left(s s^{*}\right)$.

Proof. If $t \in$ tail $(s)$ then by Lemma 3.3, $t=s r$ for some $r$ and so $t=\left(s s^{*}\right) s r$ which means that $t \in \operatorname{tail}\left(s s^{*}\right)$. Conversely, if $t \in \operatorname{tail}\left(s s^{*}\right)$ then $t=s s^{*} r$ for some $r \in S$ and so $t \in \operatorname{tail}(s)$. Hence the idempotent $s s^{*}$ is a head for tail $(s)$.

Suppose $e$ and $f$ are both idempotent heads of tail $(s)$. Then $e=f r$ for some $r \in S$ and so $e \leq f$ since $f e=f f r=f r=e$. Similar argument shows that $f \leq e$ thus $e=f$. 
Lemma 3.9. The inverse of a partial automorphism of a Cayley color graph $D_{\Delta}(S)$ is also a partial automorphism.

Proof. If $\alpha$ is a partial automorphism then

$$
\alpha^{-1}(s g)=\alpha^{-1}\left(\alpha\left(\alpha^{-1}(s)\right) g\right)=\alpha^{-1}\left(\alpha\left(\alpha^{-1}(s) g\right)\right)=\alpha^{-1}(s) g
$$

for all $s \in S$ and $g \in \Delta$.

Lemma 3.10. For all $s \in S$ the map $\alpha_{s}:$ tail $\left(s^{*}\right) \rightarrow$ tail $(s)$ defined by $\alpha_{s}(t)=s t$ is a partial automorphism of the Cayley color graph $D_{\Delta}(S)$. Furthermore, $\alpha_{s^{*}}=\alpha_{s}^{-1}$ for all $s \in S$.

Proof. It is clear that $\alpha_{s}$ maps tail $\left(s^{*}\right)$ onto tail $(s) . \alpha_{s}$ is injective since if $\alpha_{s}\left(s^{*} t\right)=\alpha_{s}\left(s^{*} r\right)$ then

$$
s^{*} t=s^{*} s s^{*} t=s^{*} \alpha_{s}\left(s^{*} t\right)=s^{*} \alpha_{s}\left(s^{*} r\right)=s^{*} s s^{*} r=s^{*} r .
$$

$\alpha_{s}$ preserves the color of the edges because $\alpha_{s}(t g)=s t g=\alpha_{s}(t) g$.

The domains of $\alpha_{s^{*}}$ and $\alpha_{s}^{-1}$ are clearly the same. If $t \in \operatorname{dom}\left(\alpha_{s^{*}}\right)$ then $t=s r$ for some $r \in S$ and so

$$
\alpha_{s^{*}}(t)=s^{*} s r=\alpha_{s}^{-1}\left(\alpha_{s}\left(s^{*} s r\right)\right)=\alpha_{s}^{-1}\left(s s^{*} s r\right)=\alpha_{s}^{-1}(s r)=\alpha_{s}^{-1}(t) .
$$

Proposition 3.11. Every partial automorphism $\alpha$ of the Cayley color graph $D_{\Delta}(S)$ is $\alpha_{s}$ for some $s \in S$.

Proof. Let $e$ be the unique idempotent head of $\operatorname{dom} \alpha$ and $s=\alpha(e)$. We show that $\alpha=\alpha_{s}$. The domain of $\alpha_{s}$ is tail $\left(s^{*}\right)=$ tail $\left(s^{*} s\right)$. We have $e \leq s^{*} s$ since

$$
e s^{*} s=\alpha^{-1}(s) s^{*} s=\alpha^{-1}\left(s s^{*} s\right)=\alpha^{-1}(s)=e .
$$

Also $s^{*} s \leq e$ since

$$
s^{*} s e=s^{*} \alpha(e) e=s^{*} \alpha(e e)=s^{*} \alpha(e)=s^{*} s .
$$

Thus, $e=s^{*} s$ and so $\alpha$ and $\alpha_{s}$ have the same domain. If $t \in \operatorname{dom} \alpha$ then $t=e r$ for some $r \in S$ and so

$$
\alpha(t)=\alpha(e r)=\alpha(e e r)=\alpha(e) e r=s t=\alpha_{s}(t) .
$$

Theorem 3.12. The set of partial automorphisms of $D_{\Delta}(S)$, with composition and inverse as the operations, is an inverse semigroup isomorphic to $S$.

Proof. By Proposition 3.11 and Lemma 3.10, the range of the map $s \mapsto \alpha_{s}$ is the set of partial automorphisms, and we have $s^{*} \mapsto \alpha_{s}^{-1}$. This map is clearly multiplicative so it remains to show that it is injective.

If $\alpha_{s}=\alpha_{t}$ then they have the same domain and so $s^{*} s$ and $t^{*} t$ are the same unique head of this domain. Thus

$$
s=s s^{*} s=\alpha_{s}\left(s^{*} s\right)=\alpha_{t}\left(s^{*} s\right)=\alpha_{t}\left(t^{*} t\right)=t t^{*} t=t .
$$




\section{The Cayley color graph of a groupoid}

In this section $\mathcal{G}$ denotes a groupoid and $\Delta$ a set of generators of $\mathcal{G}$.

Definition 4.1. Let $\Delta$ be a set of generators of the groupoid $\mathcal{G}$. The Cayley color graph $D_{\Delta}(\mathcal{G})$ of $\mathcal{G}$ with respect to the generating set $\Delta$ is the color digraph with vertices $\mathcal{G}$ and edges

$$
E=\left\{(x, z, x z) \mid x \in \mathcal{G}, z \in \Delta,(x, z) \in \mathcal{G}^{(2)}\right\}
$$

such that the edge $e=(x, z, x z)$ connects $x$ to $x z$ and has color $z$.

Example 4.2. If $A=\{a, b\}$ then the transitive groupoid $\mathcal{G}=A \times \mathbf{Z}_{2} \times A$ has eight elements. $\Delta=\{(b, 0, a),(a, 1, b)\}$ is a generating set. The Cayley color graph has two strongly connected components:

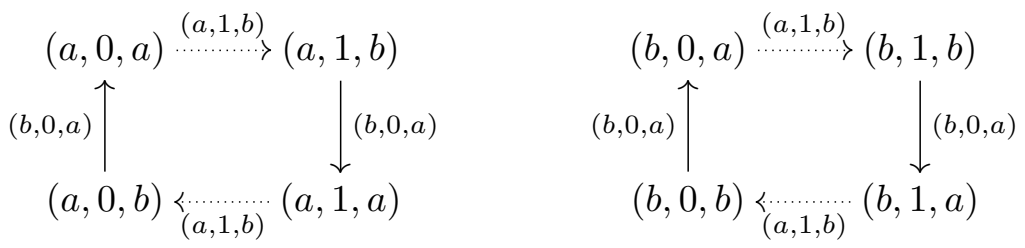

The proof of the following two lemmas are similar to that of Lemma 3.3 and Lemma 3.8 respectively.

Lemma 4.3. If $x$ is a vertex of $D_{\Delta}(\mathcal{G})$ then tail $(x)=\{x y \mid y \in \mathcal{G}\}$.

Lemma 4.4. If $x$ is a vertex of $D_{\Delta}(\mathcal{G})$ then $r(x)$ is the unique unit of $\mathcal{G}$ for which tail $(x)=$ tail $(r(x))$.

Note that every tail is strongly connected and every element of a tail is a head of the tail.

Definition 4.5. A partial automorphism of a Cayley color graph $D_{\Delta}(\mathcal{G})$ is a bijection $\alpha$ between two tails of $D_{\Delta}(\mathcal{G})$, such that $\alpha(x z)=\alpha(x) z$ for all $(x, z) \in \mathcal{G}^{(2)}$ and $z \in \Delta$.

Note that since $(x, d(x)) \in \mathcal{G}^{(2)}$ we have $(\alpha(x), d(x)) \in \mathcal{G}^{(2)}$ and so $d(\alpha(x))=d(x)$ for all $x \in \operatorname{dom}(\alpha)$. We are going to use this fact in the proof of Proposition 4.9.

The proof of the following three lemmas are similar to that of Lemma 3.7, Lemma 3.9 and Lemma 3.10 respectively.

Lemma 4.6. If $\alpha$ is a partial automorphism of $D_{\Delta}(\mathcal{G})$ then $\alpha(x y)=\alpha(x) y$ for all $x \in \operatorname{dom} \alpha$ and $(x, y) \in \mathcal{G}^{(2)}$.

Lemma 4.7. The inverse of a partial automorphism of a Cayley color graph $D_{\Delta}(\mathcal{G})$ is also a partial automorphism. 
Lemma 4.8. For all $x \in \mathcal{G}$ the map $\alpha_{x}: \operatorname{tail}\left(x^{-1}\right) \rightarrow$ tail $(x)$ defined by $\alpha_{x}(y)=x y$ is a partial automorphism of the Cayley color graph $D_{\Delta}(\mathcal{G})$. Furthermore, $\alpha_{x^{-1}}=\alpha_{x}^{-1}$ for all $x \in \mathcal{G}$.

Proposition 4.9. Every partial automorphism $\alpha$ of the Cayley color graph $D_{\Delta}(\mathcal{G})$ is $\alpha_{x}$ for some $x \in \mathcal{G}$.

Proof. Let $u$ be the unique unit of the domain of $\alpha$ and $x=\alpha(u)$. We show that $\alpha=\alpha_{x}$. The domain of $\alpha$ is the same as the domain of $\alpha_{x}$ since

$$
\operatorname{dom}\left(\alpha_{x}\right)=\operatorname{tail}\left(x^{-1}\right)=\operatorname{tail}\left(x^{-1} x\right)=\operatorname{tail}(d(x))=\operatorname{tail}(d(u))=\operatorname{tail}(u)=\operatorname{dom}(\alpha) .
$$

If $y \in \operatorname{dom}(\alpha)$ then $y=u y$ and so

$$
\alpha(y)=\alpha(u y)=\alpha(u) y=x y=\alpha_{x}(y) .
$$

Theorem 4.10. The set of partial automorphisms of $D_{\Delta}(\mathcal{G})$, with composition and inverse as the operations, is a groupoid isomorphic to $\mathcal{G}$.

Proof. By Proposition 4.9 and Lemma 4.8 the range of $x \mapsto \alpha_{x}$ is the set of partial automorphisms, and we have $x^{-1} \mapsto \alpha_{x}^{-1}$. This map is clearly multiplicative so it remains to show that it is injective.

If $\alpha_{x}=\alpha_{y}$ then they have the same domain and so $x^{-1} x$ and $y^{-1} y$ are the same unique unit in this domain. Thus

$$
x=x x^{-1} x=\alpha_{x}\left(x^{-1} x\right)=\alpha_{y}\left(y^{-1} y\right)=y y^{-1} y=y .
$$

Definition 4.11. Let $E$ be a digraph with vertices $E^{0}$ and edges $E^{1}$. If $c: E^{1} \rightarrow \mathcal{G}$ is a function where $\mathcal{G}$ is a groupoid then the skew product graph $E \times{ }_{c} \mathcal{G}$ is the digraph whose vertex set is $E^{0} \times \mathcal{G}$ and edge set is $\left\{(e, x): e \in E^{1}, x \in \mathcal{G}, x c(e) \in \mathcal{G}^{(2)}\right\}$. If $e$ is an edge from $v$ to $w$, then the edge $(e, x)$ connects $(v, x)$ to $(w, x c(e))$.

The group version of the following example can be found in [GT, Theorem 2.2.3].

Example 4.12. Let $\Delta=\left\{z_{1}, \ldots, z_{n}\right\}$ be a set of generators of $\mathcal{G}$. Let $E$ be the bouquet of $n$ directed loops with a single vertex $E^{0}=\{u\}$ and edges $E^{1}=\left\{e_{1}, \ldots, e_{n}\right\}$. If $c\left(e_{i}\right)=z_{i}$ for all $i$ then the skew product graph $E \times_{c} \mathcal{G}$ is isomorphic to the underlying digraph of the Cayley color graph $D_{\Delta}(\mathcal{G})$. The isomorphism $\alpha: E \times_{c} \mathcal{G} \rightarrow D_{\Delta}(\mathcal{G})$ is given by the vertex map $\alpha(u, x)=x$ and edge map $\alpha\left(e_{i}, x\right)=\left(x, z_{i}, x z_{i}\right)$.

\section{The Groupoid of COLOR PERMUting PARTIAL AUtomorphisms}

In $[\mathrm{FFY}]$ it is shown that the group of color permuting automorphisms of the Cayley color graph of a group $G$ is the semidirect product of $G$ and a subgroup of Aut $G$. We prove a similar result for transitive groupoids. In this section $\mathcal{G}$ denotes a transitive groupoid and $\Delta$ a set of generators of $\mathcal{G}$. 
Definition 5.1. A color permuting partial automorphism of a Cayley color graph $D_{\Delta}(\mathcal{G})$ is a bijection $\alpha$ between two tails of $D_{\Delta}(\mathcal{G})$ and a permutation $\rho$ of $\Delta$, such that $\alpha(x z)=$ $\alpha(x) \rho(z)$ for all $(x, z) \in \mathcal{G}^{(2)}$ and $z \in \Delta$.

Note that a color permuting partial automorphism is color preserving if $\rho$ is the identity permutation.

Let $H=\{\pi \in$ Aut $\mathcal{G} \mid \pi(\Delta)=\Delta\}$ be the group containing the automorphisms of $\mathcal{G}$ preserving $\Delta$. Let $\iota: H \rightarrow$ Aut $\mathcal{G}$ be the canonical embedding. Recall [Ren] that the semidirect product $\mathcal{G} \times{ }_{\iota} H$ is the groupoid $\mathcal{G} \times H$ where

$$
\left(x_{1}, \pi_{1}\right)\left(x_{2}, \pi_{2}\right)=\left(x_{1} \pi_{1}\left(x_{2}\right), \pi_{1} \pi_{2}\right)
$$

whenever $x_{1}$ and $\pi_{1}\left(x_{2}\right)$ are composable, and

$$
(x, \pi)^{-1}=\left(\pi^{-1}\left(x^{-1}\right), \pi^{-1}\right) .
$$

Lemma 5.2. For all $(x, \pi) \in \mathcal{G} \times_{\iota} H$ the map $\alpha_{(x, \pi)}:$ tail $\left(\pi^{-1}\left(x^{-1}\right)\right) \rightarrow$ tail $(x)$ defined by $\alpha_{(x, \pi)}(y)=x \pi(y)$ is a color permuting partial automorphism $\alpha_{(x, \pi)}$ of $D_{\Delta}(\mathcal{G})$. Furthermore, $\alpha_{(x, \pi)^{-1}}=\alpha_{(x, \pi)}^{-1}$ for all $(x, \pi) \in \mathcal{G} \times{ }_{\iota} H$.

Proof. $\alpha_{(x, \pi)}$ is clearly a bijection. The restriction $\rho=\pi \mid \Delta$ of $\pi$ is a permutation of $\Delta$. If $z \in \Delta$ and $(y, z) \in \mathcal{G}^{(2)}$ then

$$
\alpha_{(x, \pi)}(y z)=x \pi(y z)=x \pi(y) \pi(z)=\alpha_{(x, \pi)}(y) \rho(z) .
$$

The domains of $\alpha_{(x, \pi)^{-1}}$ and $\alpha_{(x, \pi)}^{-1}$ are clearly the same. If $y \in \operatorname{dom}\left(\alpha_{(x, \pi)^{-1}}\right)$ then $y \in$ tail $(x)$ and so $y=x x^{-1} z$ for some $z \in \mathcal{G}$ which implies

$$
\alpha_{(x, \pi)^{-1}}(y)=\pi^{-1}\left(x^{-1}\right) \pi^{-1}\left(x x^{-1} z\right)=\pi^{-1}\left(x^{-1} z\right)=\alpha_{(x, \pi)}^{-1}\left(x x^{-1} z\right)=\alpha_{(x, \pi)}^{-1}(y) .
$$

Note that $r\left(\pi^{-1}\left(x^{-1}\right)\right)=\pi^{-1}\left(x^{-1} x\right)$ is also a head for the domain of $\alpha_{(x, \pi)}$.

Proposition 5.3. Every color $\rho$-permuting partial automorphism $\alpha$ of $D_{\Delta}(\mathcal{G})$ is $\alpha_{(x, \pi)}$ for some $(x, \pi) \in \mathcal{G} \times{ }_{\iota} H$.

Proof. Let $u$ be the unique unit of the domain of $\alpha$ and $x=\alpha(u)$. To define the automorphism $\pi \in H$, suppose that $y \in \mathcal{G}$ and write $y=z_{1} \cdots z_{n}$ for some $z_{1}, \ldots, z_{n} \in \Delta$. Since $\mathcal{G}$ is transitive, there is a $t \in \operatorname{dom}(\alpha)$ such that $t y \in \operatorname{dom}(\alpha)$. The definition $\pi(y)=\rho\left(z_{1}\right) \cdots \rho\left(z_{n}\right)$ does not depend on the choice the generators $z_{1}, \ldots, z_{n}$ because if $y=w_{1} \cdots w_{m}$ for some $w_{1}, \ldots, w_{m} \in \Delta$, then

$$
\begin{aligned}
\rho\left(z_{1}\right) \cdots \rho\left(z_{n}\right) & =\alpha(t)^{-1} \alpha(t) \rho\left(z_{1}\right) \cdots \rho\left(z_{n}\right)=\alpha(t)^{-1} \alpha\left(t z_{1} \cdots z_{n}\right) \\
& =\alpha(t)^{-1} \alpha\left(t w_{1} \cdots w_{m}\right)=\alpha(t)^{-1} \alpha(t) \rho\left(w_{1}\right) \cdots \rho\left(w_{m}\right) \\
& =\rho\left(w_{1}\right) \cdots \rho\left(w_{m}\right) .
\end{aligned}
$$

It is clear that $\pi$ is an automorphism of $\mathcal{G}$ and that $\pi(\Delta)=\Delta$.

We show that $\alpha=\alpha_{(x, \pi)}$. Since $\alpha(u u)=\alpha(u) \pi(u)=x \pi(u)$, we have $d(x)=\pi(u)$. The domain of $\alpha_{(x, \pi)}$ is the tail of $\pi^{-1}\left(x^{-1} x\right)=\pi^{-1}(d(x))=u$ which is the domain of $\alpha$. If $y \in \operatorname{dom} \alpha$ then $y=u y=u z_{1} \cdots z_{n}$ for some $z_{1}, \ldots, z_{n} \in \Delta$ and so

$$
\alpha(y)=\alpha\left(u z_{1} \cdots z_{n}\right)=\alpha(u) \rho\left(z_{1}\right) \cdots \rho\left(z_{n}\right)=x \pi(y)=\alpha_{(x, \pi)}(y) .
$$


Theorem 5.4. The set of color permuting partial automorphisms of $D_{\Delta}(\mathcal{G})$, with composition and inverse as the operations, is a groupoid isomorphic to the semidirect product $\mathcal{G} \times \iota H$.

Proof. By Proposition 5.3 and Lemma 5.2, the map $(x, \pi) \mapsto \alpha_{(x, \pi)}$ is the set of color permuting partial automorphisms, and we have $(x, \pi)^{-1} \mapsto \alpha_{(x, \pi)}^{-1}$. This map is multiplicative because if $\left(x_{1}, \pi_{1}\right)$ and $\left(x_{2}, \pi_{2}\right)$ are composable then the domain of $\alpha_{\left(x_{1}, \pi_{1}\right)\left(x_{2}, \pi_{2}\right)}=$ $\alpha_{\left(x_{1} \pi_{1}\left(x_{2}\right), \pi_{1} \pi_{2}\right)}$ is the tail of $\left(\pi_{1} \pi_{2}\right)^{-1}\left(d\left(x_{1} \pi_{1}\left(x_{2}\right)\right)\right)=\pi_{2}^{-1}\left(x_{2}^{-1} x_{2}\right)$ which is the domain of $\alpha_{\left(x_{1}, \pi_{1}\right)}$. Also if $y$ is in this domain then

$$
\begin{aligned}
\alpha_{\left(x_{1}, \pi_{1}\right)\left(x_{2}, \pi_{2}\right)}(y) & =\alpha_{\left(x_{1} \pi_{1}\left(x_{2}\right), \pi_{1} \pi_{2}\right)}(y)=x_{1} \pi_{1}\left(x_{2}\right) \pi_{1}\left(\pi_{2}(y)\right) \\
& =x_{1} \pi_{1}\left(x_{2} \pi_{2}(y)\right)=\alpha_{\left(x_{1}, \pi_{1}\right)}\left(\alpha_{\left(x_{2}, \pi_{2}\right)}(y)\right) .
\end{aligned}
$$

It remains to show that our map is injective. If $\alpha_{(x, \pi)}=\alpha_{(y, \xi)}$ then they have the same domain and so $\pi^{-1}\left(x^{-1} x\right)=\xi^{-1}\left(y^{-1} y\right)$. Thus

$$
x=x \pi\left(\pi^{-1}\left(x^{-1} x\right)\right)=\alpha_{(x, \pi)}\left(\pi^{-1}\left(x^{-1} x\right)\right)=\alpha_{(y, \xi)}\left(\xi^{-1}\left(y^{-1} y\right)\right)=y \xi\left(\xi^{-1}\left(y^{-1} y\right)\right)=y .
$$

Also, if $z \in \mathcal{G}$ then $t z \in \operatorname{dom}\left(\alpha_{(x, \pi)}\right)$ for some $t \in \mathcal{G}$ and so

$$
\begin{aligned}
\pi(z) & =\pi(t)^{-1} x^{-1} x \pi(t) \pi(z)=\left(\alpha_{(x, \pi)}(t)\right)^{-1} \alpha_{(x, \pi)}(t z) \\
& =\left(\alpha_{(y, \xi)}(t)\right)^{-1} \alpha_{(y, \xi)}(t z)=\xi(t)^{-1} y^{-1} y \xi(t) \xi(z) \\
& =\xi(z) .
\end{aligned}
$$

Example 5.5. If $\mathcal{G}=\left\{x, x^{-1}, x x^{-1}, x^{-1} x\right\}$ is the trivial groupoid with two units and the generating set is $\Delta=\left\{x, x^{-1}\right\}$ then $H$ is isomorphic to $\mathbf{Z}_{2} . \mathcal{G} \times_{\iota} H$ is isomorphic to the groupoid $A \times \mathbf{Z}_{2} \times A$ of Example 4.2. The Cayley color graph of $\mathcal{G}$ has two components:

$$
x^{-1} x \stackrel{x^{-1}}{x} \underset{x}{\stackrel{x}{x}} x^{-1} \quad x \stackrel{x^{-1}}{\longleftarrow} \underset{x}{\longleftarrow} x x^{-1}
$$

\section{REFERENCES}

[Bro] R. Brown, From groups to groupoids: a brief survey, Bull. London math. Soc. 19(1987), 113-134.

[FFY] M.L. Fiol, M.A. Fiol, J.L.A. Yebra, When the arc-colored line digraph of a Cayley colored digraph is again a Cayley colored digraph, Ars Combinatoria 34(1992), $65-73$.

[GT] J.L. Gross, T.W. Tucker, Topological graph theory, Wiley Interscience Series in Discrete Mathematics and Optimization, (1987)

[Muh] P.S. Muhly, CBMS Lecture Notes, Texas Christian University, May 1990.

[Pet] M. Petrich, Inverse semigroups, John Wiley \& Sons, New York, 1984.

[Ren] J.N. Renault, A groupoid approach to $C^{*}$-algebras, Lecture Notes in Mathematics, Vol 793, Springer-Verlag, New York, 1980.

[Whi] A.T. White, Graphs, Groups and Surfaces, North-Holland publishing company, Amsterdam, 1973.

Email: nandor.sieben@nau.edu

Department of Mathematics, Northern Arizona University, Flagstaff, AZ 86011-5717 